a large number of Compton-thick AGNs in the local universe, although a correct estimate of their number density is difficult. In particular, little is known about the number density of Compton-thick AGNs at higher redshifts. Theories predict that in the early universe mergers of galaxies ${ }^{4}$ triggered rapid black-hole growth along with star formation under heavy obscuration by dust. In fact, recent studies ${ }^{5}$ show evidence that some populations of distant star-forming galaxies contain Compton-thick AGNs, whose number density may be larger than that of Compton-thin AGNs.

To constrain the average number density of Compton-thick AGNs in the universe, the absolute intensity of the XRB at $30 \mathrm{keV}$ is a critical parameter. Even if their cosmological evolution is unknown, we can still discuss it within the population synthesis models of the $\mathrm{XRB}^{6}$. From the number density of Compton-thin AGNs, revealed by X-ray surveys below $10 \mathrm{keV}$, we can estimate their contributions to the XRB above $10 \mathrm{keV}$ by integrating the fluxes over redshift. In this calculation, an averaged broadband spectrum of Compton-thin AGNs must be assumed. The missing intensity of the XRB around $30 \mathrm{keV}$ is then attributed to the integrated emission from Compton-thick
AGNs. For instance, by assuming a constant number ratio with respect to Compton-thin AGNs, one can 'indirectly' estimate the number density of Compton-thick ones.

In fact, the XRB intensity and spectrum over the 3-50 keV band had been thought to be well established by the all-sky mission, HEAO-1, 27 years ago ${ }^{7}$. However, after the 1990s, there have been many claims that the HEAO-1 result may be significantly (by 20-40\%) underestimated when compared with the XRB spectrum obtained by modern imaging X-ray satellites below $10 \mathrm{keV}$. To settle this important issue, Frontera and co-workers ${ }^{2}$ have made an independent measurement of the XRB spectrum in the 15-50 keV band with a non-imaging instrument onboard the BeppoSAX satellite, using a different technique from the HEAO-1 experiment. Rather unexpectedly, they find the XRB intensity at $30 \mathrm{keV}$ to be perfectly consistent with the original HEAO-1 result, with an upper bound of $12 \%$ higher than the best estimate by HEAO-1. A similar result has also been reported by Chrazov and co-workers ${ }^{8}$ using the INTEGRAL satellite. The authors infer a present mass density of SMBHs to be $\sim 3 \times 10^{5}$ solar masses per $\mathrm{Mpc}^{3}$ as the result of accretion, including that occurring in Compton-thick AGNs. This value is somewhat smaller than previous estimates?

Of course, this approach for estimating the number density of Compton-thick AGNs from the XRB depends on the following: the true absolute intensity of the hard XRB, which is uncertain at the $10-20 \%$ level; the average broadband spectra of Compton-thin AGNs; and the cosmological evolution of Compton-thick AGNs, which is assumed to be the same as that of Compton-thin AGNs. To finally solve the problem, we must wait for more sensitive hard-X-ray imaging missions above $10 \mathrm{keV}$, such as NeXT, Simbol-X and XEUS, which will enable us to directly detect the Compton-thick AGNs that constitute a significant fraction of the peak intensity of the XRB.

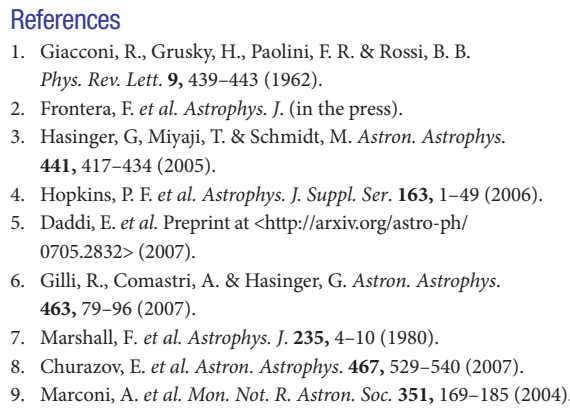

\title{
PIERRE-GILLES DE GENNES
}

\section{Across the divides}

Theoretical physicist, experimentalist, teacher, public personality - Pierre-Gilles de Gennes, who died on 18 May 2007, wore all of these labels, and more. His rare ability to flow across boundaries between scientific disciplines led him to found the area of 'soft matter', for which he was awarded the Nobel Prize in 1991. But his work on polymers and liquid crystals was just one aspect of his research interests.

Born in 1932 in Paris, he attended École Normale Supérieure in 1951 and worked in Saclay on magnetic phase transitions, using neutron scattering, between 1955 and 1959, during which he obtained his $\mathrm{PhD}$. Then after a post-doc at Berkeley and service in the French navy, he set up a research group on superconductors at the Université de Paris in Orsay.

In hindsight, it's clear that the framework to describe phase transitions in magnets and superconductors should apply more generally to any transitions between ordered and disordered states in other complex systems such as polymer solutions and liquid crystals. But before de Gennes made the connection, they were considered disparate fields. He saw simplicity and order within complex, disordered and soft systems. Not one to rest on his laurels, he moved on to study interfaces, wetting and adhesion. His fascination with how water adheres to some surfaces but not others eventually helped wine producers to improve pesticide coverage of a grape. Then after his retirement from the Collège de France, he focused on problems that were more biological; his most recently published paper (March 2007) is entitled "Collective neuronal growth and self organisation of axons".

Aside from his research output, he published several books aimed at a broader audience. He was an ideal mentor. Following his Nobel prize, he visited more than 150 schools across France to encourage students to study science, showing them the beauty of hands-on, everyday physics. $\mathrm{He}$ was also supportive of his fellow scientists and actively participated in the peer-review process. Unsurprisingly, his was a name often recommended by authors to review their work, across several fields of research.

As an example of his approach to science, de Gennes did not set out to create



the now ubiquitous flat-screen display whose technology is based on his work: he simply thought liquid crystals posed an interesting theoretical problem and used his intuitive methods to study them. Thanks to his legacy, we will never forget to do research for the sake of it, without being preoccupied with application, and regardless of the discipline.

May Chiao 